\title{
Are We Getting to All That Matters? The Need for Perceptual Guidance.
}

\author{
Anstein Gregersen
}

\begin{abstract}
Congenital deafblindness (CDB) profoundly affects perception of the environment. CDB can affect both which objects are perceived, and which features of the objects are experienced. In order to gain better access to the environment and the social world, it could therefore potentially be beneficial if a child with CDB was perceptually directed by a seeing and hearing person. However, such a form of intervention is not unproblematic. This article argues that such a perceptual guidance is only acceptable if the leading in perception is agency sensitive and contains three features: that of joint perception; a culturally sensitive organization of perceptual fields, and a dynamic back-and-forth mode of leading and exploring.
\end{abstract}

\section{Keywords}

Congenital deafblindness, tactile perception, communication, conversational dyad 


\section{Introduction}

How can children ${ }^{1}$ with congenital deafblindness (CDB) access and partake in their environment? Perception, of course, is the gateway to the environment and one of the profound effects of CDB is the multiple ways in which it impacts perception. One example is that many objects ${ }^{2}$ are outside a child with CDB's tactile reach and are therefore not perceived. Another example is that the child might not experience what encultured seeing and hearing peers consider the salient features of the objects. Yet another example is the inimical effect of CDB on perception of social interactions that often are too fast paced and perceptually complex for tactile exploration. These, and other, challenges one might think, are impetus for designing interaction forms where the partner perceptually shows the child the environment, call it "perceptual guidance".

In the literature on deafblindness, however, we find good reasons for being sceptical to such directing of perception (McInnes 1999, Nafstad and Rødbroe 2007, Souriau and Brede, 2008). Instead we find a focus on creating access through communication (see e.g. Hartmann 2012, Janssen, M. and Rødbroe, I, 2007, Marková, 2016, Nafstad \& Rødbroe 2015, Souriau 2009), and especially language (Souriau 2009). This does not, these days, imply a demand for persons with CDB to learn and express themselves in "culturally expected forms" (Nafstad, 2014, p.60). The field recognises diversity in persons with CDB's exploration and communicative efforts. This recognition of diversity tells against directing a child with CDB's perception since the child's exploratory needs might differ greatly from the seeing and hearing partner's culturally similar way of exploring.

So, on the hand we have impetus for designing interactions where the partner shows the child how to perceive the environment. On the other hand, there are important objections to directing perceptual exploration.

Thus, it seems we are at an impasse. The overall concern of this article is to elaborate this tension. Furthermore, on the basis of this elaboration I extract what it would take for the tension to abate. This would demand balancing leading and following where agency sensitivity and joint perception are crucial ingredients. I further develop and set out a model of perceptual guidance in this article's companion piece "Getting to What Matters: A Model of Perceptual Guidance" (Gregersen 2020).

\section{Congenital Deafblindness and the Perceptual Field}

How is it that CDB affects environmental perception? This is a very complex question and a full answer would require a discussion of a whole host of factors that would take us beyond the scope of this article. We can, however, get a sense of some of 
the challenges that $\mathrm{CDB}$ poses by considering some of the contributions of sight and hearing. ${ }^{3}$ Scholarly treatment of deafblindness often calls sight and hearing for "distance senses". This is strictly speaking not correct. Touch, for instance, can discern at distance as well (Fulkerson, 2012). Moreover, sight and hearing give access to more of the environment than just objects at distance. However, with a thicker description of sight and hearing than that of a 'distance' sense only, we can nevertheless capture some of the challenges that CDB poses. Before giving a fuller - though far from exhaustive description, I should note that my analysis addresses the situation of a child with no functioning sight or hearing. This does not include all children with $\mathrm{CDB}$, some of whom still have some residual sight or hearing, but some main elements of the description should still hold for them.

The phenomenologist Hans Jonas described sight in terms of three characteristics: " (1) simultaneity in the presentation of a manifold, (2) neutralization of the causality of sense-affection, (3) distance in the spatial and mental senses" (1954, p. 507). These characteristics are displayed for example in the case of a sighted child playing in a sandbox. The child swiftly perceives the sandbox, the toys in it and the other children playing (1). It sees all this from a distance (3). And the causality behind the sense-affection (2) is neutralized because with sight there is no direct contact. This is easiest explained by comparing it with touch: In touching the sandbox, the child is potentially directly and bodily affected in a pleasant or un-pleasant manner. With sight, however, the child is freer to consider the environment and to decide if he wants to engage it directly with his bodily surfaces. ${ }^{4}$

Analyzing the relation between the child with CDB's perceptual field and the environment considering Jonas' description will reveal some of the main consequences of CDB on experience.

First, due to the limited range of tactile perception it is not likely that the child with CDB discovers that there is play in the sandbox. For the most part, objects at larger distances are not perceived without sight and hearing. A benefit of sight is also that it presents the perceiver with a large manifold at distance quickly. It is not possible for a child with CDB to perceive all objects in his surroundings since tactile exploration is time consuming and mentally demanding. This has ramifications for the partner and leaves two options: Either not even attempt to engage the larger environment. Or, we acknowledge when going out in the world that the partner must select which objects the child possibly should engage with. Seeing children will often attend to the adult's objectdirected visual gaze in order to orient themselves (e.g. Csibra, \& Johnson, 2008), in other words, they let the adult play a guiding role in what is socially significant and what to attend to. Nafstad and Rødbroe (2007) also acknowledge that partners must make significant aspects of the world accessible for discovery. This, in my terminology, is a 
form of perceptual guidance: A perceptual guidance in selecting which objects to perceive. Perceptual guidance can here aid in perceptual economy and the child can offload the perceptual work of finding the significant objects to the partner. Thereby the child can instead concentrate on perceiving these objects.

Secondly, even if objects are within the perceptual field many objects are not in the perceptual field in a manner that allows the child to experience what the objects are. Here is an example from the sociologist R. Michalko, who has acquired blindness. He recalls standing at the top of a stairwell leading down to a subway station. After a rush of people had walked up the stairs Michalko walks down slowly, guided by his right hand and feet. The following happens:

Suddenly I touched something with my left knee. It was a gentle touch, but a touch nonetheless. Just as suddenly, the voice of a woman: "No, out of his way. Come over here." The touch I felt was a child! The anxiety that rushed through my body froze me. I had almost knocked a child down two flights of stairs. There was no question now. I needed help. (1999, p. 11.)

Michalko perceived a touch through touch. The content of the tactile perception is "a gentle touch, but a touch nonetheless". Through touch Michalko perceived that there is a living creature that has touched him, but it is only with hearing the woman's voice that he perceives the situation in its graveness: The object becomes the child, and the content of the perception is a child that is almost knocked "down two flights of stairs". The lack of visual perception renders Michalko's tactile perception of the child inadequate. To borrow a suitable formulation from Immanuel Kant: a child with CDB will often experience its perceptual field as a "rhapsody of perceptions" (1997, p. A156/B195. For further discussion see Gregersen, 2018). This leads to difficulty in experiencing and cognizing objects. To return to a similar example; that of children playing in a sandbox, here the play is complex and often fast-changing. This makes it incredibly hard for a child with CDB to perceive and interact even though the play occurs within the child's perceptual field. The unstableness of the former's perceptual field is tied to the fact that tactile perception, often, is much more time demanding than sight and contains less manifold. I have previously argued (Gregersen 2018) that the partner can to some extent stabilize the child's perceptual field by aligning herself bodily with the child. This bodily alignment can therefore be part of perceptual guiding.

Thirdly, when an object is within a child with CDB's perceptual field in a manner that allows for tactile exploration the child will not necessarily experience the same as a seeing and hearing child. When we experience an object as something - say, we experience a toy-gun as a toy-gun - the object contains a manifold. In taking the toy-gun 
as a toy-gun some of the manifold of the toy-gun are taken to be the significant ones. These culturally salient features are brought to fore, while others recede to the back. Objects often contain possibilities for different kinds of experiences and what features of the manifold we attend to is important for the experiences we have. Here are two examples involving my son Kasper who was born deaf and blind with no-light perception and who was most likely completely deaf from birth. ${ }^{5}$

Kasper would often play with a toy-gun where the barrel was a speaker that gave off a fart-sound when the trigger was pulled. Nevertheless, I cannot really imagine that Kasper knew he was playing with a toy-gun. He seemed mostly interested in the shape and feel of the gun, and not it's uses in human interactions. There is nothing wrong in that, but it also means that he did not experience the cultural meaning of the object. Another example is that Kasper sometimes would light up in a big smile when one of his siblings came crying. To bring his attention to the fact that his sibling was crying we needed to explicitly show this. If we had let him explore on his own, he would probably first attend to other features, or manifold, than those of his sibling's tears. And this would also entail a temporal dimension. His attention to the tears would probably be too late. Too late for the sibling to experience empathy and too late for Kasper to show empathy. With perceptual guidance, however, we can offer the child possibilities of a culturally sensitive way of experiencing the environment. This gives the child better conditions for social comprehension and interaction. Moreover, it will enrich him in experiences.

\section{Two Arguments Against Directing Perception}

There are, then, potential benefits to directing perception. But, nevertheless, Nafstad and Rødbroe issues a clear warning against it:

There is a risk that we stop the exploration of the child because we think it lasts too long or looks repetitive. This is the same as not giving time and space for image formation. To try to make the child explore in more culture-similar ways, or not at all because it looks strange and repetitive, is of course not what we really want to do. To show him the world by directing his touch is the same as directing somebody's visual perception. This is not what we want. We do it when we are not aware of the way he explores. The child first has to discover the world in his own way, the task is to make some important aspects of the world available for exploration and discovery. Important aspects are for instance the persons (and 
animals) in his family; these are much more important to discover than any other “object". (2007, p.19)

There is indeed a high risk of not acknowledging the particular way in which children with CDB perceptually explore the world (Souriau and Brede, 2008).

Our first argument against directing perception is a combination of a theoretical and empirical argument. Relating back to the discussion of how congenital deafblindness affects perception it is reasonable to say that how the child with CDB experiences the world is often very different from how their seeing and hearing peers do. Additionally, although I described how CDB affects perception, of course not all children with CDB experience the same way: Rather through tactile encounters with the world, a very particular life-story unfolds - in a more distinct manner than perhaps is the case with seeing and hearing children (Marková, 2016). Take this to be a partial explanation for the need of recognizing diversity among the population with CDB.

Given that a child with CDB's experiences hang together somewhat differently than for seeing and hearing peers, instruction in how to perceive from partners might be quite problematic. There might occur dis-congruence between the child and the partner in how and what they experience. The child might not find the partner's exploratory strategies comprehensible and significant, or at worst loses trust in his partners and withdraw from interactions. Add into this that there is often low readability of the child's exploratory and communicative efforts. This hinders him in negotiating with his interaction partner and then fronting his own interests and understanding of the world. Reactiveness to the child's bodily tactile expressions should therefore take priority in interventions.

Another argument against directing perception can be constructed from the historical development in deafblindstudies where directing and instruction into language is no longer a preferred interaction form. So, this second argument is an argument from converging practice.

Both historically and currently deafblind studies has been mainly concerned with communication (see e.g. Hartmann 2012, Janssen, M. and Rødbroe, I, 2007, Marková, 2016, Naftstad \& Rødbroe 2015, Souriau 2009). The history of deafblind education is sometimes divided into four periods (See Nafstad and Johansen 2018, also Hart 2006). Starting with a first period between 1800 and 1950, we find success stories, such as the education of Helen Keller, Laura Bridgman and Olga Skorokhodova. These remarkable individuals learned to use conventional language in "spoken, signed, finger-spelled and written form" (Hart, 2006, p. 264). The teacher transferred conventional language, understood as a skill residing in the world, through touch so that the persons with CDB adopted much of the same language that their seeing and hearing peers used. In the 
second period, starting with the advent of the rubella children, "Relationship, resonance, and co-active movement" (Hart, 2006, p. 264) become more central. The goal was still to develop symbolic communicative skills that resembled seeing and hearing people's communicative skills. However, this rarely was the outcome: Few examples emerged of a person with deafblindness declaring experiences, sharing feelings, memories and wishing (Rødbroe and Souriau 2000). The explanation for this, according to Rødbroe and Souriau (2000) is that communication was understood as deliverance of messages, quite like how Hart describes teaching in the first period. Also, in the third period (Nafstad and Johansen 2018), starting in the 1980's, deafblind studies conceived language as something that resides outside of people and must be picked up and learned through instructions. Turning to present time Nafstad and Johansen characterize the fourth period by the recognition of diversity in communication (2018). Sense-making and communication emerge from transactions between people. This leads gradually to language as being more co-constructed from transactions between people than an acquired skill (Souriau and Brede, 2008). Today, interactions begin with recognizing and responding to idiosyncratic bodily communicative expressions and they do not entail instruction in cultural symbolic language.

As we can see, the internal developments in deafblind studies have moved the field away from a concern with instruction into pre-conceived forms of experiencing and communicating about the world. Rather interactions should be in a reactive environment (See e.g. McIness 1999, Nafstad and Rødbroe 2015, Nicholas, Johannessen and van Nunen 2019). This tells a cautionary tale against too stringent forms of perceptual guidance.

These are genuine insights. However, my diagnosis is that, on the one hand, the developments in deafblindstudies, or at least significant parts of it, have shied too far away from perceptually directing the child with CDB. Simultaneously, and on the other hand, they are also still too indebted to the conversational dyad (premised on the seeing person) as the way to create reactiveness. Thereby not addressing conditions for a focus on a broader field of perception and exchange.

My argument and focus below addresses both problems. To illustrate this, I will look at two prominent and influential conceptions of interaction in the literature.

\section{The Conversational Dyad, the Intervenor and Conditions for Experience}

Our short recount of deafblind studies occupation with communication shows that there have been major changes in the conception of language and how partners should relate to children with $\mathrm{CDB}$. However, the form of the interaction has remained 
much the same: It entails a conversational dyad where two people exchanges meaningful tactile expressions face-to-face (Naftstad \& Rødbroe 2015, Souriau 2009). Sometimes there are three people in the conversation, creating a group situation with a more complex turn-taking that is more perceptually demanding. But it still is about conversing. While the conversational dyad is in many situations the right form of interaction a worry is that it does not sufficiently address the full impact that CDB has on experience. I have previously argued (Gregersen 2018) that it is not optimal for stabilizing perception, sometimes in practice cluttering the child's perceptual field rather than making it comprehensible. Relatedly and as emphasized in this article, the conversational dyad does not always give the best possible access to the larger environment. In arguing this I will simplify the conversational dyad; these interactions are more varied than what I will portray them as. Still the arguments will bring out a challenge about accessing the environment.

In the description of how CDB affects perception we saw that it created challenges for both the selection of objects the person with CDB perceives, as well as whether he perceives the culturally salient features of the objects. And this latter point relates to the content of the experiences. If for instance the child does not know how to perceive and interact with children playing in a sandbox, this will prevent him from experiencing play with peers. Since sight has the great benefit of perceiving a manifold swiftly, a partner could potentially be a good guide into such play. As a dyad concerned with conversation, however, this form of interaction does not really address how to perceive and interact with rapidly changing situations like play. And it is sometimes assumed that it is impossible for a child with CDB to play with peers (e.g. Rødbroe \& Souriau 2000).

Notice that the conversational dyad is compatible with co-exploration. The child can, of course, initiate exploration, before the partner reactively joins in (e.g. Nicholas, Johannessen and van Nunen 2019), but this will not remedy the need for leading in perception by a sighted and hearing partner.

The effect of not having access to social situations is that the dyad can create a very local culture on the margins of the broader culture, thereby restricting the kind of experiences and cognitive development available for the child.

Another related problem of social cognition with the conversational dyad is that there is in these interactions a stark imbalance in legibility. The recognition of diversity in the child with CDB's sense-making and communication implies that a major task for the partner is to read the child's communicative efforts. That, of course, implies understanding the child's perspective that underlies his communication: e.g. what experiences do his expressions emerge from? Observation before and while engaging with the child is therefore important for a proper interaction. Deafblind studies today 
even use, successfully, video-analyses for this purpose. In the conversational dyad the partner has access through sight and hearing to the child - fine-tuned through videoanalyses. The child, however, has much less access to the partner and her context.

Both the lack of access to fast-changing social interactions and the legibility of partners creates risks for dependency on a few partners for communicating and understanding the world. Or put differently, the interpretive framework that the child develops is based on prolonged interaction with a few significant partners, but the access to the partners perspective is very low. That will make the child's understanding of the environment, most significantly his partners, very contextual. At the same time communication will also, then, be very contextual: a few partners become master interpreters of the child. The risk is then an epistemic and communicative imbalance that creates an undesired dependency.

These arguments simplify real interactions in the conversational dyad but bring out a possible problematic consequence. The conversational dyad is still a significant form of interaction, but the arguments point towards potential benefits of conceiving a form of interaction complementary to the conversational dyad that gives children with CDB experience and engagement with the environment.

Another prominent conception of interactions in the literature is McInnes' "intervenor". The intervenor is concerned with perceptual access to the environment: "Intervention" is defined as "the PROCESS that takes place between the person who is deafblind and the person providing support, in such a way that the disability caused by the loss of the effective use of the distance senses of sight and hearing will be minimized." (1999, p.76). McInnes further states that: "Intervention is a process, the purpose of which is to enable the deafblind person to establish and maintain maximum control over his or her environment at a level appropriate to physical ability and level of functioning." (1999, p.76).

The intervenor creates access to people, activities and things (what I for shorthand have called "objects"). McInnes likens the intervenor to a person who simultaneously provides the services of a phone operator, a taxi driver and a tourist information center worker (1999): A phone operator enables communication with other people, but does not decide whom you should call, when, why, or whether it is in your best interest to make the call. The taxi driver does not decide your destination, the time of departure, nor your travel companions, but transports you in a safe and efficient manner. A tourist information center clerk can inform you about the places of interest, but it is you who in the last instance decides if you follow the clerk's recommendations. McIness' laudable ambition is that the intervenor should support the child with CDB so that he can maintain maximum control and make informed decisions. McInnes' intervenor is close to perceptual guidance in the analogy with a combined tourist 
information center clerk and taxi driver. However, in McIness' job-description, the taxi driving tourist information clerk is restricted to suggesting which objects to perceive and securing mobility.

The intervenor model seems as fitting designs for many interactions. The question is if it is sufficient? For McInnes the intervenor is concerned with suggesting objects for the child's exploration. But he does not address the challenge that the perceptual field might be changing too fast for perceptions to form, bringing out the need for perceptual guidance. Social interactions are perhaps the most important example of where the perceptual field is changing rapidly. Secondly, McInnes understates the need for creating access to culture. As argued, we must also address if the culturally salient features are perceived.

This relates to McInnes' ambition that the child with CDB should be able to make informed decisions. In this we find an argument for reactiveness in that it can secure autonomy for the child with CDB. However, to be autonomous and make informed decisions about what objects he wants to engage with the child must first know what to decide amongst. So, the child must understand what the objects are or what they can provide.

Another way of putting this point is that while a reactive environment is crucial, there is also a need for creating conditions that allows the person with CDB to perceive his environment, partners included. This means that partners cannot only be reactive, they must also guide into the world in a way that makes perceptual engagement possible. In some cases, this is about knowing how to perceive complex situations, like with children playing, where the complexity comes from the fact that objects in the situation changes fast. In other cases, it is about knowing the role that objects have in the human environment, like with knowing how to perceive that smooth thing made of wood as a table. Our environment is largely a social environment where objects are what they are in terms of their cultural role.

\section{Criteria for Perceptual Guidance}

If directing someone's perception implies instructing them into cultural-similar ways of exploring, then there are indeed strong arguments against directing a child with CDB's perceptions. However, interactions that solely rely on reactiveness do not sufficiently address the challenges that CDB poses for experience. So, it seems we have reached an impasse. We need to perceptually direct, but we cannot. The varied ways in which persons with CDB experiences makes it desirable with directing perception, but the varied needs for exploration makes cultural similar directing of perception undesirable. 
Perhaps there is a way out of this impasse in striking the right balance between leading and reactiveness. Perceptual guidance can be neither straightforward instructions into cultural similar forms of exploration nor merely reactive coexploration. On this middle road perceptual guidance would have to be a creative process of joint perception where the partner is also, at times, leading the exploration. The creativeness lies in the effort to find ways of co-perceiving where the child can pickup the way the partner perceives, while at the same time there is room for the child's own exploratory needs.

So, a model of perceptual guidance must first of all be agency sensitive. Three further features seem necessary. First, partner and child must jointly perceive. Secondly, the partner must offer the child a culturally sensitive organization of the perceptual fields. Thirdly, there must be a dynamic back-and-forth mode of leading and exploring between partner and child.

We can spell out these features a little further. In joint perception child and partner not only just perceive the same object, they also attune to each other's exploration. The partner enables this by showing the child possible ways of organizing their overlapping perceptual fields. This perceptual organization occurs both in the selection of objects for perception, as well as in how and what to perceive. This showing is culturally sensitive, enabling the child to understand the culture-like way of comprehending the object, while taking into concern the child's exploratory needs. The child follows the partner because he trusts the partner to reveal the environment and culture in a manner that he can perceive and understand. Knowing that he also can lead and explore in his own way. The partner remains open and receptive to this agency on the child's part.

\section{Conclusion}

The profound impact of CDB on perception reveals itself not just in terms of distal perception, but also in access to social interactions and experiencing the cultural salient features of objects. Perception, even if one thinks ultimately that language is more important, should therefore be an important topic in deafblind studies. Central contributions to deafblind studies stress reactiveness to the child's exploration. I have in this article argued that there is also a need for leading in perception, or perceptual guidance. In order to evaluate further if perceptual guidance is a viable alternative interaction form there remains both theoretical and empirical work to be done.

Empirically, one could study instances of perceptual leading in interactions forms that we find in the everyday. There are probably many examples and situations in which perceptual guidance takes place without an agent that operates with an explicit model 
undergirding such actions. Empirical studies should look whether such guiding, or leading, reveals voluntary directing, creates agency and enables access to an environment that the child finds both comprehensible and interesting.

We also need theoretical work that develops perceptual guidance and takes a stance on whether it is justifiable. I have argued that we need to strike a balance between reactiveness and leading in guidance, on this we need a model that makes the dynamic back-and-forth in exploration central. Guidance means that the child at times follows the lead of the partner, but it must also be a dynamic interaction where the child leads and can co- create the conditions for joint- perception. I develop such a model in Gregersen (2020), in effect arguing that perceptual guidance can be justified theoretically. 


\section{References}

Dammeyer, J. (2014). “Deafblindness: A review of the literature”. Scandinavian Journal of Public Health, 42(7), 554-562. https://doi.org/10.1177/1403494814544399

Fulkerson, M. 2012. “Touch Without Touching”. Philosphers Imprint. Vol. 12, NO. 5, PP. 1-15. http://hdl.handle.net/2027/spo.3521354.0012.005

Gregersen, A. (2018). "Body with Body: Interacting with Children with Congenital Deafblindness in the Human Niche". Journal of Deafblindstudies on Communication. Vol.4, pp.67-83. Groningen. University of Groningen Press. https:// doi.org/10.21827/jdbsc.4.31374

Gregersen, A. (2020). "Getting to What Matters: A Model of Perceptual Guidance”. Submitted to Journal of Deafblindstudies on Communication. Groningen. University of Groningen Press.

Hart, P. (2006), “Using imitation with congenitally deafblind adults: establishing meaningful communication partnerships". Inf. Child Develop., 15:

263-274. doi:10.1002/icd.459

Elizabeth S. Hartmann (2012) A Socio-cognitive Approach to How Children with Deafblindness Understand Symbols, International Journal of Disability, Development and Education, 59:2, 131-144, DOI: 10.1080/1034912X.2012.676373

Janssen, M. and Rødbroe, I. (eds.). (2007). Communication and Congenital Deafblindness. II: Contact and Social Interaction. St. Michielsgestel: VCDBF/Viataal.

Jonas, H. (1954). "The Nobility of Sight: A Study in the Phenomenology of The Senses" Philosophy and Phenomenological Research, Vol.14(4), pp.507-519.

Kant, I. (1997). Critique of Pure Reason. Trans. Paul Guyer and Allen Wood. The Cambridge Edition of the Works of Immanuel Kant. New York: NY. Cambridge University Press.

Marková, I. (2016). The Dialogical Mind. Common Sense and Ethics. Cambridge: England. Cambridge University Press.

McInnes, J. M. (1999). “Intervention.” in A Guide to Planning and Support for Individuals Who Are Deafblind. McInnes, John M (ed.). Toronto: Canada. University of Toronto Press.

Moll, H., Arellano, D, Guzman, A., Cordova, X., Madrigal, J.A. (2015). 'Preschoolers' mutualistic conception of seeing is related to their knowledge of the pronoun "each other"'. Journal of Experimental Child Psychology, Volume 131, PP. 170-185. ISSN 0022-0965. https://doi.org/10.1016/j.jecp.2014.11.006. 
Moll, H., Khalulyan, A. (2017) “"Not See, Not Hear, Not Speak”: Preschoolers Think They Cannot Perceive or Address Others Without Reciprocity'. Journal of Cognition and Development, 18:1, 152-162, DOI: 10.1080/15248372.2016.1243116

Michalko, R. (1999). The Two in One. Philadelphia: PA. Temple University Press.

Nafstad, A. V. (2014). “Optimizing Interaction: A Precondition for Assesing Cognition” in Ask Larsen, F. \& Damen, S. (ED.) Guidelines for Assessment of Cognition in Relation to Congenital Deafblindness. Stockholm: Nordic Center for Welfare and Social Issues.

Nafstad, A. V. and Johansen, K. (2018). “Fire epoker i døvblindfeltetes historie” in Ed. Lid, I.M. Diakoni og Velferdsstat. Oslo: Norway. Gyldendal Akademisk.

Nafstad, A. V. and Rødbroe, I. B. (2015). Communicative Relations. Interventions that create communication with persons with congenital deafblindness. Statped Sørøst, Fagavdeling døvblindhet/kombinert syns- og hørselsvansker. Aalborg: Denmark. Materialcenteret.

Nafstad, A.V. and Rødbroe, I.B. (2007). "Co-creating Communication with Persons with Congenital Deafblindness". PP.17-23. Communication Network Update Series, Number 8. Nordic Staff Training Centre for Deafblind Services. https://nordicwelfare.org/wpcontent/uploads/2017/10/CNUS_08_web.pdf

Nicholas, J. T., Johannessen, A. M., and van Nunen, T. (2019). Tactile Working Memory Scale - A Professional Manual. Nordic Welfare Centre. https://nordicwelfare.org/wpcontent/uploads/2019/12/NWC-TWMS-Handbok-webb.pdf

Rødbroe, I., \&Souriau, J. (1999). “Communication” in A Guide to Planning and Support for Individuals Who Are Deafblind. McInnes, J. M. (ed.). Toronto: Canada. University of Toronto Press.

Senju A., Csibra G., Johnson M.H. (2008) “Understanding the referential nature of looking: Infants' preference for object-directed gaze" in Cognition Volume 108, Issue 2. https://doi.org/10.1016/j.cognition.2008.02.009

Souriau, J. (2009). “Deafblindness, language, culture and identity: A dialogical perspective”. „30 Jahre Erziehung und Förderung von Menschen mit Hörsehbehinderung/Taubblindheit in Würzburg“. Colloque Würzburg. No source.

Souriau, J. and Brede, K.S. (2008). "Language and Congenital Deafblindness".

Communication Network Updates Series, No 10, NVC. https://nordicwelfare.org/wpcontent/uploads/2017/10/CNUS10_web.pdf 


\section{Acknowledgements}

I would like to thank Rosemarie Van Den Breemer, Kåre Letrud and Anne V. Nafstad.

Anstein Gregersen, Chair of Department and Associate Professor of Philosophy, Inland School of Business and Social Sciences/ Department of Law, Philosophy and International Studies, Inland Norway University of Applied Sciences; email: < anstein.gregersen@inn.no>

${ }^{1}$ In line with the focus on early communication and support for social interaction that we find in many North-American and European countries (Dammeyer, 2014) I will be concerned with children with CDB. Parts of the discussion is likely applicable to other groups as well.

2 For the sake of brevity, I will in the following use "object" to encompass things that can be perceived, such as material objects, persons and events.

${ }^{3}$ I will not disucss here the important role of sight and hearing in mutual recognition between perceivers. See Moll, H., Arellano, D, Guzman, A., Cordova, X., Madrigal, J.A. (2015) and Moll, H., Khalulyan, A. (2017). Also, I will not address perception of language.

4 This explains one of the benefits of the white cane. It provides extended reach, but also a neutralization of the causality of sense-affection since the object is perceived through the white cane. The cane, importantly, is not the body.

${ }^{5}$ By his first year he had bi-lateral CI and enjoyed sounds and understood quite a bit of verbal communication. In addition to using conventional signs he also learned to speak a few words. 